\title{
Isolation and genetic characterization of canine adenovirus type 2 from a domestic dog showing neurological symptoms
}

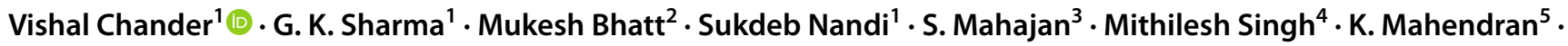

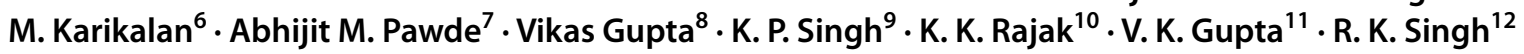

Received: 3 November 2020 / Accepted: 7 June 2021 / Published online: 14 June 2021

(c) Sociedade Brasileira de Microbiologia 2021

\begin{abstract}
Canine adenoviruses (CAVs) are of two types: canine adenovirus type 1 (CAV-1), which causes infectious canine hepatitis, and canine adenovirus type $2(\mathrm{CAV}-2)$, which is mainly associated with the respiratory type of disease in dogs. Due to the widespread use of modified live vaccines to control canine adenoviral infections and subsequently reduced disease incidence, CAVs are often neglected by clinicians. Although a number of studies are available about CAV-1 prevalence in India, only meagre information is available about CAV-2. This study reports the CAV-2 infection in a vaccinated dog with neurological and respiratory symptoms which was found negative for other canine pathogens like canine distemper virus and canine parvovirus. The virus was successfully isolated from rectal swab in MDCK cells and characterized by immunofluorescence assay and virus neutralization test. On phylogenetic analysis of partial E3 region, the Indian CAV-2 grouped in a separate clade different from established subgroups. An insertion of "G" nucleotide was reported at nucleotide (nt.) position 1077 in the E3 gene of Indian CAV-2 isolates which led to a frameshift in the coding region of E3 gene thereby imparting additional eleven amino acids to its $\mathrm{C}$-terminal end in comparison to isolates from other parts of the world. This may have an implication on the functional role of $\mathrm{E} 3$ protein inside the cell. This study reinforces the unique signature insertion in the E3 gene of Indian CAV-2 and is the second study in the world to report the association of CAV-2 with neurological disease in dogs.
\end{abstract}

Keywords Canine adenovirus $2 \cdot$ Neurological symptoms $\cdot$ Dogs $\cdot$ India

Responsible Editor: Fernando R. Spilki

Vishal Chander

drvishal1@gmail.com

$\square$ V. K. Gupta

gupta.drvivek@gmail.com

1 Virology Laboratory, Centre for Animal Disease Research and Diagnosis (CADRAD), ICAR-Indian Veterinary Research Institute (IVRI), Izatnagar, Bareilly, Uttar Pradesh 243122, India

2 ICAR Research Complex for NEH Region, Sikkim Centre, Tadong, Gangtok, Sikkim 737102, India

3 Division of Veterinary Biotechnology, ICAR-IVRI, Izatnagar, Bareilly, Uttar Pradesh 243122, India

4 Immunology Section, ICAR-IVRI, Izatnagar, Bareilly, Uttar Pradesh 243122, India

5 Referral Veterinary Polyclinic, ICAR-IVRI, Izatnagar, Bareilly, Uttar Pradesh 243122, India
6 Centre for Wildlife, ICAR-IVRI, Izatnagar, Bareilly, Uttar Pradesh 243122, India

7 Division of Veterinary Surgery, ICAR-IVRI, Izatnagar, Bareilly, Uttar Pradesh 243122, India

8 CCS National Institute of Animal Health, Baghpat, Uttar Pradesh 250609, India

9 Pathology Laboratory, CADRAD, ICAR-IVRI, Izatnagar, Bareilly, Uttar Pradesh 243122, India

10 Divison of Biological Products, ICAR-IVRI, Izatnagar, Bareilly, Uttar Pradesh 243122, India

11 CADRAD, ICAR-IVRI, Izatnagar, Bareilly, Uttar Pradesh 243122, India

12 ICAR-IVRI, Izatnagar, Bareilly, Uttar Pradesh 243122, India 


\section{Introduction}

Canine adenovirus (CAV), a non-enveloped dsDNA virus, is a member of the genus Mastadenovirus under the family Adenoviridae. Based on genetic and antigenic characteristics, canine adenoviruses are divided into two types: canine adenovirus type 1 (CAV-1) and canine adenovirus type 2 (CAV-2) [1]. Although CAV-1 affects the digestive tract and causes infectious canine hepatitis (ICH) accompanied by uveitis and interstitial nephritis in dogs, CAV-2 is mainly associated with respiratory type of disease [2]. Currently, the control of canine adenoviral disease in canids is mainly based on the use of live attenuated vaccines containing attenuated strains of CAV-2, which also crossprotects against CAV-1 [3]. However, besides the widespread use of vaccines to prevent adenovirus infection in canids, recurrent CAV-1- or CAV-2-associated infections have been documented in dogs and many wildlife species raising the concern about the real epidemiological picture of canine adenovirus in dogs [4-7].

Although CAV-2 is supposed to cause a transient and asymptomatic or mild form of respiratory disease and remains restricted to the upper respiratory tract causing laryngeotracheitis, in presence of other pathogens or in immune-compromised host, it has been reported to cause severe necrotising bronchitis and interstitial pneumonia [3]. CAV-2 is also associated with fatal cases of diarrhoea $[8,9]$ and neurological disease in dogs [5]. With the widespread use of modified-live vaccines to protect dogs from infectious agents such as canine adenovirus, it is now being considered as a pathogen of less importance and is neglected by clinicians. Nevertheless, fatal outbreaks of CAV-2 are being reported across the globe including some more recent studies $[1,10]$. This study reports the CAV-2 infection in a dog with neurological and respiratory symptoms which was confirmed by the sequencing of the partial E3 gene and virus isolation in MDCK cells. The sequence generated in this study was also compared with the CAV-2 sequences available from different parts of the world to elucidate the genetic heterogeneity among the CAV-2 strains.

\section{Materials and methods}

\section{Sampling}

In November 2018, a Labrador retriever dog of 6 months of age from Bareilly, Uttar Pradesh, was presented with the symptoms of coughing, neurological spasms and vomit after food intake. No fever was noticed in the dog and the nasal, conjunctival and rectal swabs were collected in the viral transport media suspecting canine distemper. As these samples were collected for the purpose of disease diagnosis in clinics, therefore, no ethical approval was required. As per the history provided by the owner, vaccination was carried out with a single shot of multivalent vaccine at 3 months of age. The animal had been intensively treated with antibiotics and supportive fluid therapy but it was reported to have died the next day, as conveyed by the owner, and thus, further samples could not be collected.

\section{PCR assays and sequencing}

While nasal and conjunctival swabs were tested for the presence of canine distemper virus (CDV), the rectal swab was tested for the presence of canine parvovirus (CPV) and canine adenovirus-specific nucleic acids through polymerase chain reaction (PCR). RNA and DNA were isolated from nasal, conjunctival and rectal swabs using RNeasy Mini Kit (Qiagen, Germany) and DNeasy kit (Qiagen, Germany) according to the manufacturer's instructions. Extracted nucleic acids were subjected for PCR amplification using previously published primers for detection of CDV, CPV and CAV-1/2 [2, 11-13]. The plasmid carrying the E3 gene of CAV-1 isolate (GenBank Accession no. KX181846), Nucleocapsid gene of CDV (GenBank Accession no. MF964188) and VP2 region of CPV (GenBank Accession no. KC429662) available in our laboratory were used as a positive control in the PCR assay for respective viral agents. The amplified product was gel purified using QIA Quick Gel Extraction Kit (Qiagen, Germany) and cloned into pJET 1.2 blunt vector (Thermo Fisher Scientific) followed by sequencing (Eurofins Genomics, India).

\section{Phylogenetic analysis}

For phylogenetic reconstruction, available sequences representing the $\mathrm{E} 3$ region of $\mathrm{CAV}-2, \mathrm{CAV}-1$ and other related adenoviruses were retrieved from NCBI and aligned using online MAFFT (Multiple Alignment using Fast Fourier Transform) version 7 software (http://mafft. cbrc.jp/alignment/server/index.html) in MSA program. The best-fit nucleotide substitution model was predicted in the Molecular Evolutionary Genetics Analysis software version X (MEGA-X) [14] and the phylogenetic tree was constructed employing the Maximum-Likelihood method using Kimura 2-parameter model with 1000 bootstrap replications to confirm the authenticity of the taxa analysed for each node. 


\section{Virus isolation}

For virus isolation, filtered supernatant of the rectal swab was inoculated onto a monolayer of Madin-Darby Canine Kidney (MDCK) cells in $25 \mathrm{~cm}^{2}$ tissue culture flasks and incubated at $37^{\circ} \mathrm{C}$ for $1 \mathrm{~h}$. After $1 \mathrm{~h}$ of adsorption, Minimum Essential Medium Eagle (Sigma-Aldrich, USA) containing $10 \%$ foetal bovine serum along with 10,000 units penicillin, $10 \mathrm{mg}$ streptomycin and $25 \mu \mathrm{g}$ amphotericin B per millilitre (Antibiotic Antimycotic Solution, $100 \times$ Sigma-Aldrich, USA) was added and the flask was incubated at $37^{\circ} \mathrm{C}$. The cells were examined daily under the microscope and blind passaged until visible CPE was evident.

\section{Fluorescent antibody test (FAT)}

To confirm the identity of isolated virus, direct fluorescent antibody test (DFAT) was performed in a 96-well cell culture plate seeded with MDCK cells (Fig. 1). The MDCK cells were inoculated with the $200 \mathrm{TCID}_{50}$ of isolated virus and incubated at $37^{\circ} \mathrm{C}$ for $96 \mathrm{~h}$. After $96 \mathrm{~h}$, the cells were washed with PBS and fixed with chilled acetone/PBS (phosphate-buffered saline; 80:20) for $30 \mathrm{~min}$ at $4{ }^{\circ} \mathrm{C}$. Thereafter, acetone/PBS solution was discarded and anti-CAV-2 polyclonal antiserum conjugated to fluorescein isothiocyanate (Canine adenovirus FITC conjugated; VMRD, Pullman, Washington) was added to the virus-infected and control wells followed by incubation at $37^{\circ} \mathrm{C}$ for $30 \mathrm{~min}$. After incubation, the plate was washed several times with FA rinse buffer (pH 9.0) and observed under a fluorescent microscope after adding the mounting fluid.

\section{Virus neutralization assay}

The identity of the isolated virus was also confirmed by virus neutralization test (VNT). In brief, the MDCK-adapted virus $\left(200 \mathrm{TCID}_{50} / \mathrm{ml}\right)$ was incubated with an equal volume of anti-CAV-2 antibody serum for $1 \mathrm{~h}$ at $37{ }^{\circ} \mathrm{C}$ and inoculated in 96-well plate seeded with MDCK cells followed by incubation at $37^{\circ} \mathrm{C}$ for $96 \mathrm{~h}$. The VNT plate was observed under the microscope $96 \mathrm{~h}$ post-inoculation to record the results.
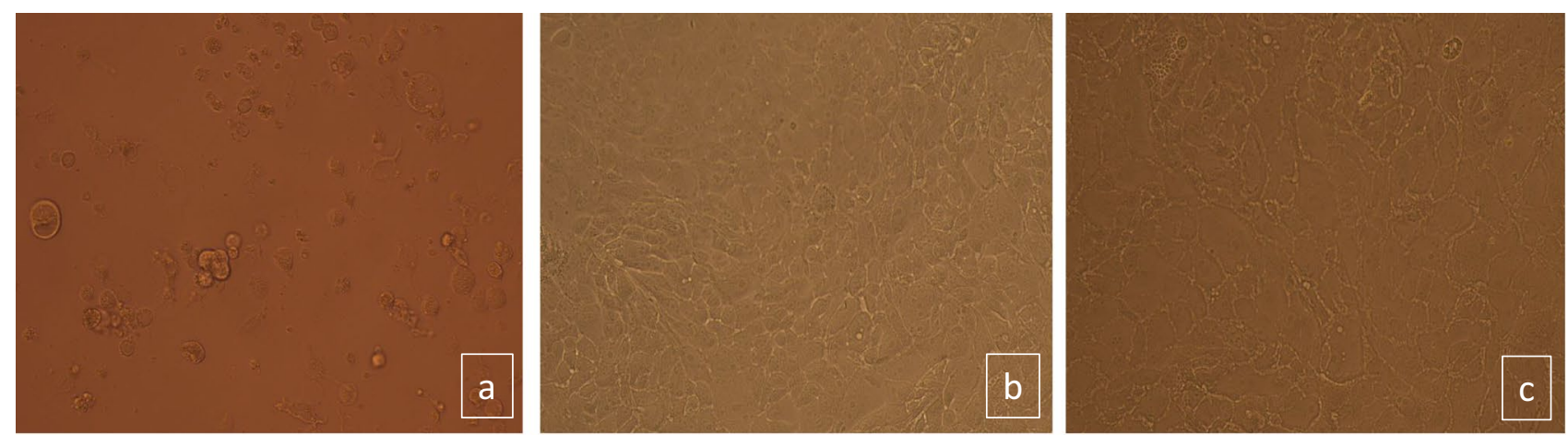

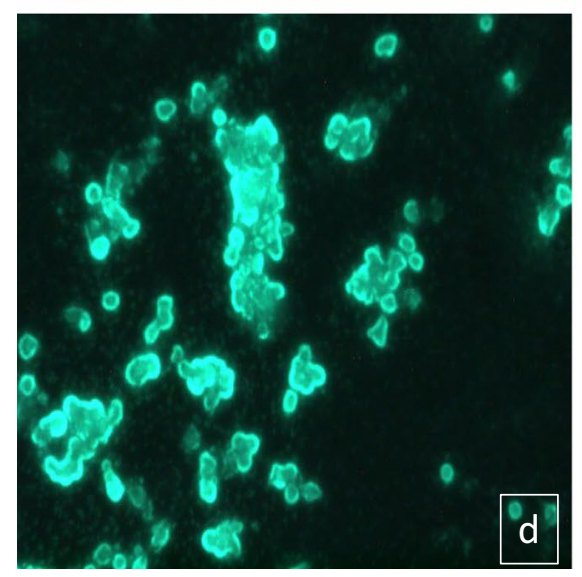

CAV 2 in MDCK

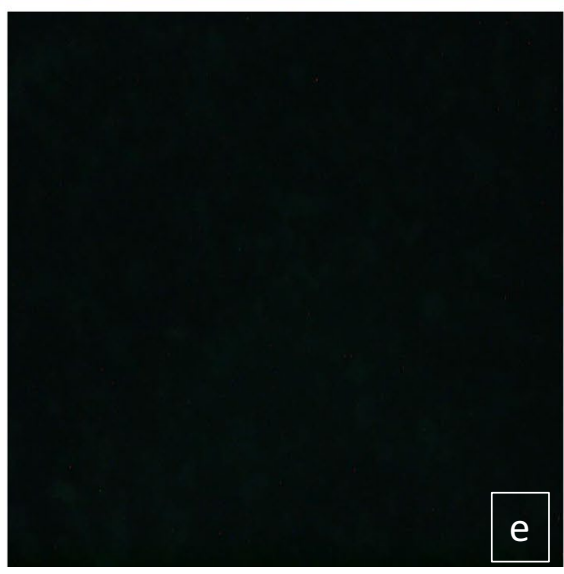

MDCK mock infected

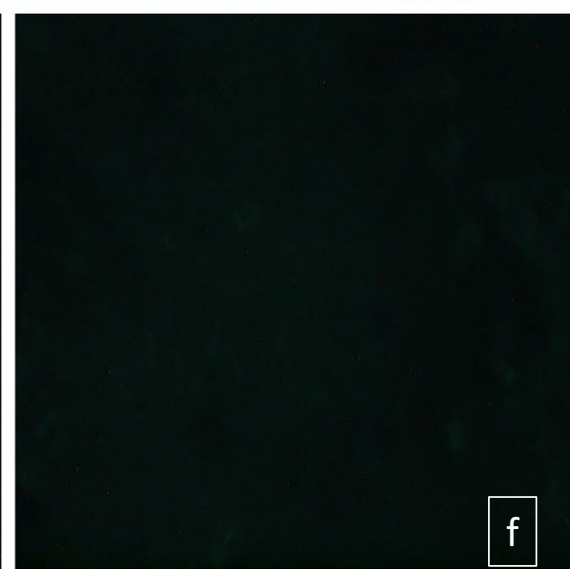

VNT in MDCK
Fig. 1 Virus isolation in MDCK Cell line: a Infected monolayer of MDCK cells shows cell rounding, detachment, cytoplasmic fusion, vacuolation and cell death at 3 dpi $(20 \times$ magnification $)$. b Mockinfected MDCK cells at 3 dpi $(20 \times)$. c VNT in MDCK cell line showing clear neutralization with no observable CPE $(20 \times)$. d Direct
FAT (fluorescent antibody test) showing MDCK cells infected with $10^{-1}$ dilution of CAV-2 with characteristic CPE $(20 \times)$. e Mockinfected MDCK cells at $3 \mathrm{dpi}(20 \times)$ and $\mathbf{f}$ VNT in MDCK cell line showing clear neutralization with no noticeable fluorescence $(20 \times)$ 


\section{Results and discussion}

Canine adenovirus type 2 is a common respiratory pathogen and causes a mild disease in dogs. Only occasionally, it has been reported to cause gastrointestinal or neurological complications $[5,8]$. In this study, the samples collected from a dog showing respiratory signs with nervous system involvement were tested for the presence of CDV, CPV and CAV1/2. While the samples were tested negative for CDV and CPV, an amplicon of 1032 bp length was amplified in PCR using E3 gene-based differential PCR for CAV1/2 [2, 12]. The amplified product was sequenced and 858 bp sequence representing E3 region of CAV-2 was submitted to NCBI with the accession number MT318141. Nucleotide BLAST analysis of sequence generated in this study confirmed the presence of CAV-2-specific nucleic acid in the positive sample.

The nt. alignment of CAV-2 sequence generated in this study with the other CAV-2 sequences available from different countries revealed a "G" nucleotide insertion at the nt. position 1077 in the E3 gene (ORF2) (Fig. 2a). These findings are in agreement with a recent study from India [9] and reinforce the unique signature "G" nt. insertion in E3 gene of Indian CAV-2 isolates. The addition of " $G$ " residue resulted in a frameshift and imparted additional eleven amino acids (aa) to the E3 protein of Indian CAV-2 isolates (Fig. 2b). As it is suggested that the E3 protein of $\mathrm{CAV}-2$ is crucial for modulating the host immune response in adenovirus-infected cells [15], it will be imperative to investigate the effect of a comparatively larger E3 protein on host-virus interactions and disease progression.

On phylogenetic reconstruction, while CAV-1 and CAV-2 were clustered in different groups, bat and equine adenovirus sequences formed the out-group (Fig. 3). Among the CAV-2 sequences, as was previously described [1], Chinese and Turkish strains formed a different subgroup separated from the America-Europe subgroup. The CAV-2 sequences from India including the sequence generated in this study formed a monophyletic group separated from the previously identified two subgroups of CAV-2 (Fig. 3). The comparison of the region corresponding to terminal 245 aa of putative E3 region ORF2 of canine adenovirus type 2 revealed an evolutionary divergence of $3.3 \%$ at aa level between the Indian strain and the Toronto A26/61 strain (Accession no. U77082) (Table 1) grouped under America-Europe subgroup in the phylogenetic tree. Indian CAV-2 isolated in the current study appears to be more closely related to the strains from Italy and Brazil than the Chinese and Turkish isolates of CAV-2. However, this study is based on only the partial sequence of E3 region and therefore, further studies with more sequence data are augmented to get a clear picture of the evolutionary history of CAV-2 circulating in India.
The virus was successfully isolated in MDCK cells using rectal swab found positive for CAV-2-specific nucleic acids as a source of inoculum. The visible CPE, characterized by cell rounding, detachment, cytoplasmic fusion, vacuolation and cell death, was seen as early as $72 \mathrm{~h}$ post-infection from the 5th passage onwards (Fig. 1). In DFAT, green fluorescence could be seen in the virus-infected cells while no colour signal was detected in the control wells which confirmed the presence of CAV-2 in the infected wells (Fig. 1). The results of DFAT were further validated in VNT using CAV-2-specific antisera. While CAV-2-specific CPE could be seen after $96 \mathrm{~h}$ of incubation in control wells infected with the virus, mock-infected and the test wells inoculated with virus-antibody mixture did not show any visible CPE. Further, testing of VNT plate by DFAT revealed no colour signal in test wells inoculated with virus-antibody mixture indicating complete neutralization of virus by anti-CAV-2 antibody (Fig. 1).

The detection and isolation of CAV-2 from a dog showing nervous signs which was subsequently found negative for other canine pathogens reinforce the neuronal tropism of CAV-2, as was described previously [5]. As the dog was vaccinated with the multivalent vaccine 3 months before the appearance of clinical signs, the chances of vaccine strain-induced disease are negligible. Also, a previous study has shown that dogs vaccinated with CAV-2 vaccine do not shed vaccine virus beyond the 6th day postvaccination $[16,17]$. Sequencing results also ruled out any chances of vaccine strain-induced disease in the dog. To our limitation, as the outcome of infection was fatal, studies to investigate pathological changes in the brain and other tissues could not be carried out due to the non-compliance of the dog owner.

The reason for the development of neurological signs could be assigned to the involvement of the brain or the hypoxia due to involvement of the respiratory system as suggested in a previous study where the nervous symptoms were noticed in the pups infected by CAV-2 but any appreciable changes in the nervous tissue were not observed [5]. It is to mention here that CAV-2 engages the coxsackievirus and adenovirus receptor (CAR), a member of the CTX subfamily of immunoglobulin (Ig) superfamily to transduce the motor neurons and undergo retrograde transport [18]. Soudais and co-workers reported the preferential transduction of neuron by CAV-2 following intramuscular injection [18]. Additionally, retrograde axonal transport is also used by other viruses like poliovirus and bacterial tetanus toxin which also induce nervous symptoms [19]. Although a similar mechanism of retrograde transport may be responsible for neurological disease in CAV-2-infected dogs, the association of CAV-2 with the neurological disease should be used with caution unless it is proven with sufficient experimental data. 

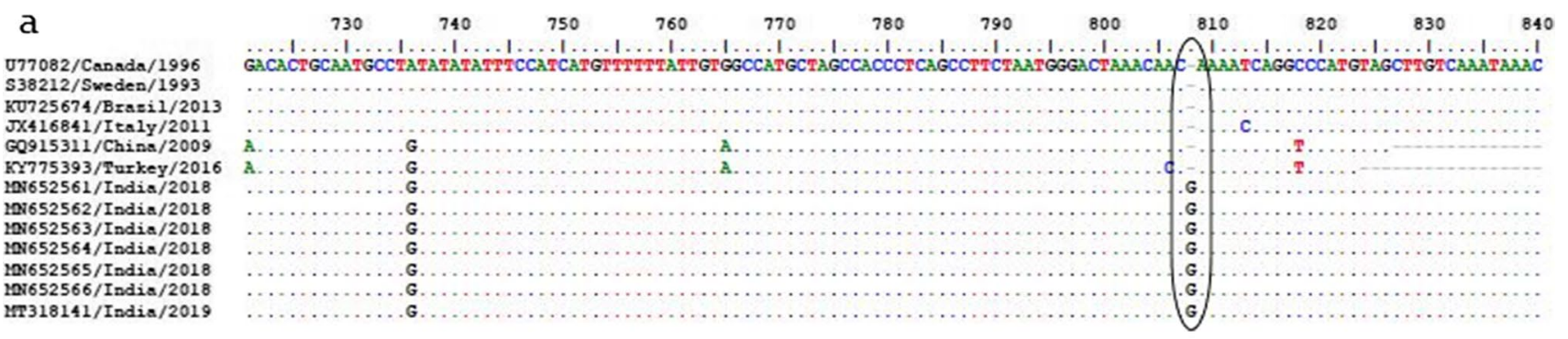

b

U77082/Canada/1996 S38212/Sweden/1993 KO725671/Brasil/2015 MF $356370 / \mathrm{Italy} / 2016$ KU725672/Brasil/2015 JX116841/Italy/2011
GQ915311/China/2009 GQ915311/China/2009
KY775393/Turkey/2016 KY775391/Turkey/2016 ins $652561 /$ India/2018 1D*652562/India/2018 nos $652563 /$ India $/ 2018$ 19x652564/India/2018 1nv652565/Indis/2018 nst652566/India/2018 Mr318141/Indis/2019

U77082/Canada/1996 S38212/Sweden/1993 KU725671/Brasil/2015 MF $356370 / I \operatorname{taly} / 2016$ KU725672/Brasil/2015 JX416841/Italy/2011 G0915311/China/2009 KY775393/Turkey/2016 KY775391/Turkey/2016 ini652561/India/2018 nni652561/India/2018 now52564/Indis/2018 108652564/India/2018
108652565/Indis/2018 10s652565/Indis/2018 1Ds652566/India/2018
UT318141/Indis/2019 077082/Canada/1996
S38212/Sweden/1993 KU725671/Brasil/2015 MF356370/Italy/2016 KU725672/Brasil/2015 JX116841/Italy/2011 GQ915311/China/2009 KY775393/Turkey/2016 KY775391/Turkey/2016 nos $652561 /$ Indin $/ 2018$ 1ns652562/Indis/2018 nox652563/India/2018 pos $652564 /$ Indis $/ 2018$ nos $652565 /$ Indis/2018 mos IT318141/India/2019

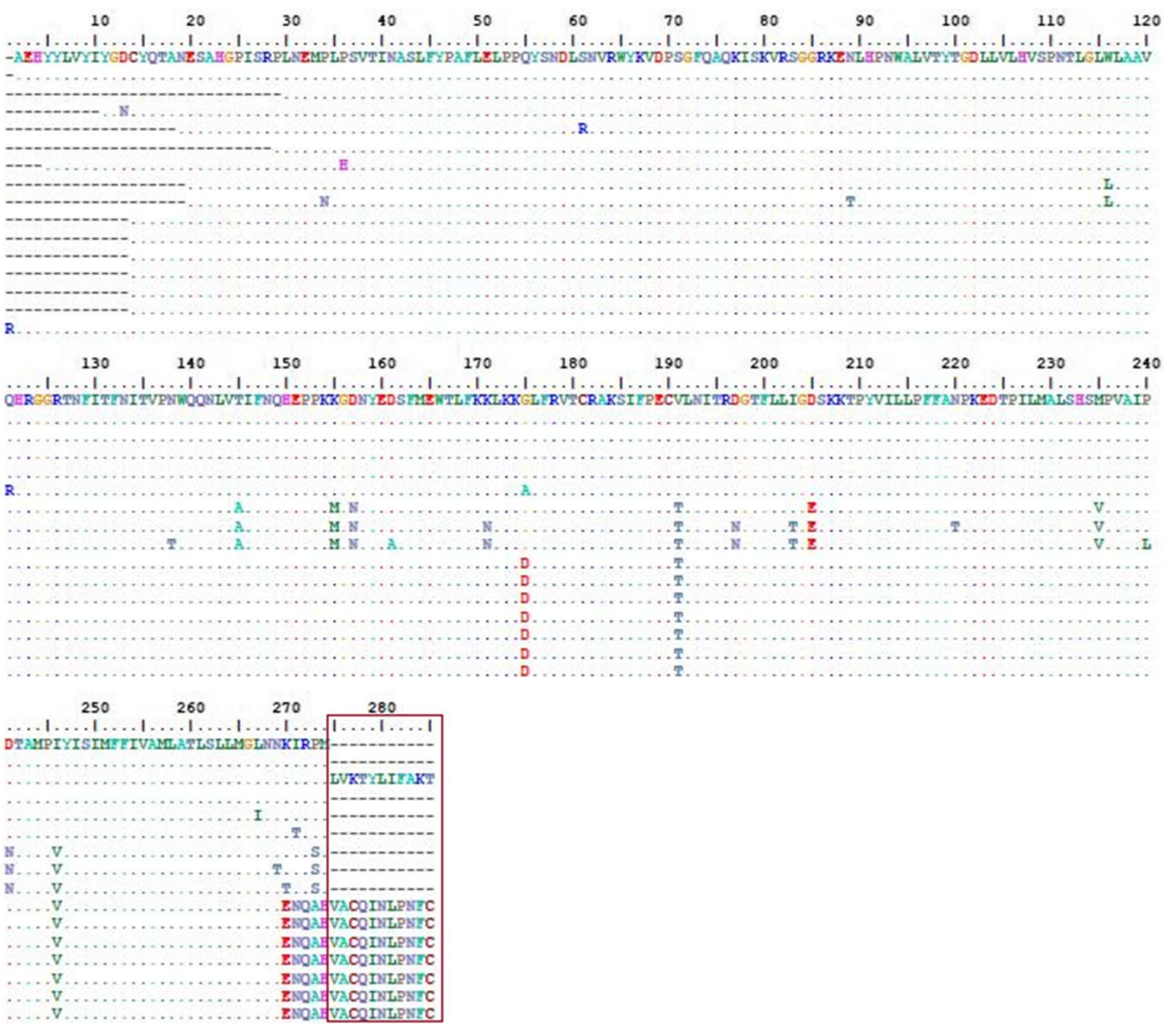

Fig. 2 Aligned nucleotide sequence of E3 region of CAV-2 generated in this study along with CAV-2 sequences from different parts of the world (a) and deduced amino acid sequence alignment of different

After the introduction of live-modified vaccines and their widespread use to control disease, the incidence of canine adenoviral infections has reduced [20] and until a recent study [9], the prevalence of CAV-2 in the dog population in India was unreported. It is to mention that there are only a few reports of CAV-2-associated neurological disease of dogs and hence, the possibility of CAV-2 causing the nervous signs is ignored by the clinicians which could
CAV-2 isolates (b). [The insertion of "G" nt. in the Indian isolates is represented by eclipse (a) and the extension of reading frame by eleven aa in the Indian isolates is indicated by a rectangle (b)]

be the reason that CAV-2 remained unreported in the past. Moreover, the clinical picture of CAV-2 overlaps with that of other canine pathogens like canine herpes virus, canine parainfluenza virus, canine distemper virus and Bordetella bronchiseptica which further complicates the diagnostic approach by the clinicians. As the dog was vaccinated 3 months back and proper vaccination schedule was not followed, there is no point of vaccine ineffectiveness although 


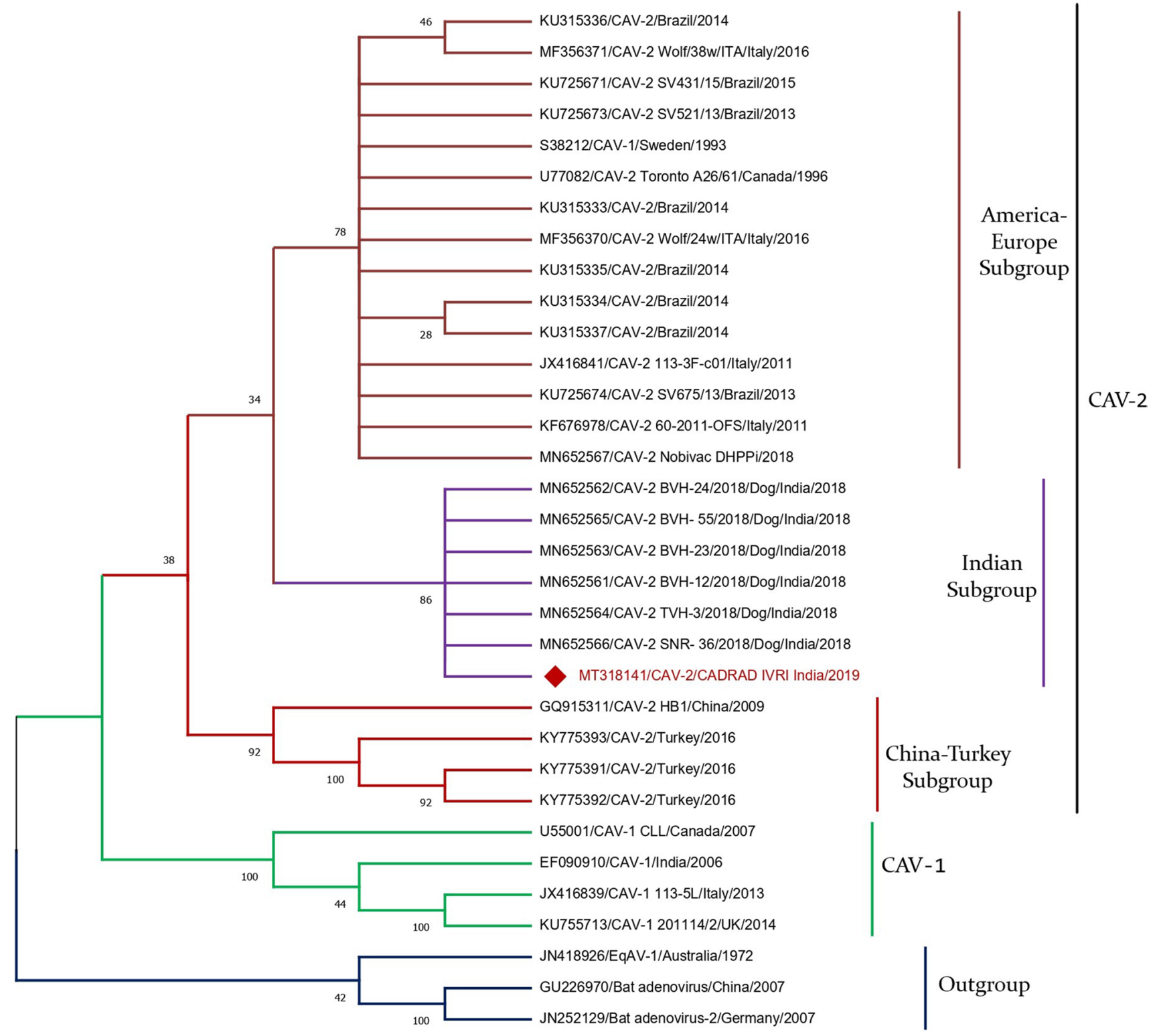

Fig. 3 Phylogenetic tree based on the sequence of partial E3 region from 33 strains of adenoviruses (CAV-1, CAV-2, EqAV-1 and Bat adenovirus) generated by using the Maximum-Likelihood method and Kimura 2-parameter model with 1000 bootstrap replications

reported previously [7, 21]. The reason for CAV-2-associated disease may be ascribed to the non-adherence of dog owners to the proper vaccination schedule and large free-roaming population of canids acting as reservoirs of infection.

\section{Conclusion}

This study could establish the presence of CAV-2 in a dog showing respiratory and nervous symptoms. The virus could successfully be isolated in MDCK cells and was using MEGA-X software. Equine and Bat adenoviruses were used as an outgroup. GenBank accession numbers are provided for all the sequences

characterized by sequencing of partial E3 region, immunofluorescence and virus neutralization test. Detection of a novel CAV-2 in cases of gastroenteritis and dog showing nervous signs from two distant locations in the country augments the need for generation of more sequence data from different geographical areas and involving the longer regions of viral genome to get a clear picture of molecular epidemiology of CAV-2 in India. The study also reinforces the importance of adherence to proper vaccination protocols in order to prevent the disease in susceptible population. 
Acknowledgements All the authors are thankful to the Director, ICARIndian Veterinary Research Institute, Izatnagar, India, for providing the funds and research facilities to carry out this work.

\section{Declarations}

Conflict of interest The authors declare no competing interests.

\section{References}

1. Timurkan MO, Aydin H, Alkan F (2018) Detection and molecular characterization of canine adenovirus type $2(\mathrm{CAV}-2)$ in dogs with respiratory tract symptoms in shelters in Turkey. Vet Archiv 88(4):467-479

2. Hu RL, Huang G, Qiu W, Zhong ZH, Xia XZ, Yin Z (2001) Detection and differentiation of CAV-1 and CAV-2 by polymerase chain reaction. Vet Res Commun 25:77-84

3. Balboni A, Verin R, Morandi F, Poli A, Prosperi S, Battilani M (2013) Molecular epidemiology of canine adenovirus type 1 and type 2 in free-ranging red foxes (Vulpesvulpes) in Italy. Vet Microbiol 162(2-4):551-557

4. Appel M, Bistner SI, Menegus M, Albert DA, Carmichael LE (1973) Pathogenicity of low-virulence strains of two canine adenovirus types. Am J Vet Res 34(4):543-550

5. BenetkaV WH, Kudielka I, Pallan C, Rothmüller G, Möstl K (2006) Canine adenovirus type 2 infection in four puppies with neurological signs. Vet Record 158(3):91-94

6. Buonavoglia C, Martella V (2007) Canine respiratory viruses. Vet Res 38(2):355-373

7. Kalinowski M, Adaszek L, Miłoszowska P, Skrzypczak M, Zietek-Barszcz A, Kutrzuba J, Gradzki Z, Winiarczyk S (2012) Molecular analysis of a fragment of gene E1B 19K of canine adenovirus 2 (CAV-2) isolated from dogs with symptoms of cough. Pol J Vet Sci 15(3):425-430

8. Macartney L, Cavanagh HM, Spibey N (1988) Isolation of canine adenovirus- 2 from the faeces of dogs with enteric disease and its unambiguous typing by restriction endonuclease mapping. Res Vet Sci 44(1):9-14

9. Ramidi A, Ganji VK, Buddala B, Yella NR, Manthani GP, Putty K (2019) E3 gene-based genetic characterization of canine adenovirus- 2 isolated from cases of canine gastroenteritis in India revealed a novel group of the virus. Intervirology 62(5-6):216-221

10. Dowgier G, Lahoreau J, Lanave G, Losurdo M, Varello K, Stella M, Ventriglia G, Bozzetta E, Martella V, Buonavoglia C, Decaro N (2018) Sequential circulation of canine adenoviruses 1 and 2 in captive wild carnivores, France. Vet Microbiol 221:67-73

11. Bhatt M, Rajak KK, Chakravarti S, Yadav AK, Kumar A, Gupta V, Chander V, Mathesh K, Chandramohan S, Sharma AK, Mahendran K, Sankar M, Muthuchelvan D, Gandham RK, Baig M, Singh RP, Singh RK (2019) Phylogenetic analysis of haemagglutinin gene deciphering a new genetically distinct lineage of canine distemper virus circulating among domestic dogs in India. Transbound Emerg Dis 66(3):1252-1267

12. Chander V, Nandi S, Verma R (2013) Detection of infectious canine hepatitis virus in vaccines by PCR. Adv Anim Vet Sci 1(6):191-193

13. Chander V, Chakravarti S, Gupta V, Nandi S, Singh M, Badasara SK, Sharma C, Mittal M, Dandapat S, Gupta VK (2016) Multiplex Amplification Refractory Mutation System PCR (ARMS-PCR) provides sequencing independent typing of canine parvovirus. Infect Genet Evol 46:59-64 
14. Kumar S, Stecher G, Li M, Knyaz C, Tamura K (2018) MEGA $\mathrm{X}$ : molecular evolutionary genetics analysis across computing platforms. Mol Biol Evol 35:1547-1549

15. Burgert HG, Blusch JH (2000) Immunomodulatory functions encoded by the E3 transcription unit of adenoviruses. In: Becker Y, Darai G (eds) Molecular evolution of viruses - past and present. Springer, Boston, pp 13-25

16. Bass EP, Gill MA, Beckenhauer WH (1980) Evaluation of a canine adenovirus type 2 strain as a replacement for infectious canine hepatitis vaccine. J Am Vet Med Assoc 177(3):234-242

17. Cornwell HJ, Koptopoulos G, Thompson H, McCandlish IA, Wright NG (1982) Immunity to canine adenovirus respiratory disease: a comparison of attenuated $C A V-1$ and $C A V-2$ vaccines. Vet Rec 110(2):27-32

18. Soudais C, Laplace-Builhe C, Kissa K, Kremer EJ (2001) Preferential transduction of neurons by canine adenovirus vectors and their efficient retrograde transport in vivo. FASEB J 15:2283-2285
19. Salinas S, Schiavo G, Kremer EJ (2010) A hitchhiker's guide to the nervous system: the complex journey of viruses and toxin. Nat Rev Microbiol 8(9):645-655

20. Abdelmagid OY, Larson L, Payne L, Tubbs A, Wasmoen T, Schultz R (2004) Evaluation of the efficacy and duration of immunity of a canine combination vaccine against virulent parvovirus, infectious canine hepatitis virus, and distemper virus experimental challenges. Vet Ther 5(3):173-186

21. Balboni A, Mollace C, Giunti M, Dondi F, Prosperi S, Battilani M (2014) Investigation of the presence of canine adenovirus (CAdV) in owned dogs in Northern Italy. Res Vet Sci 97:631-636

Publisher's note Springer Nature remains neutral with regard to jurisdictional claims in published maps and institutional affiliations. 